\title{
三维被动摆动水翼对波浪滑翔机推进 动力的性能研究*
}

\author{
胡 峰 1,2 赵文涛 $1,2,3$ 黄 琰 ${ }^{1,2}$ 俞建成 ${ }^{1,2}$ 赵宝德 ${ }^{1,2}$ 崔 健 ${ }^{1,2}$ \\ (1. 中国科学院沈阳自动化研究所机器人学国家重点实验室 沈阳 110016; \\ 2. 中国科学院机器人与智能制造创新研究院 沈阳 110016; \\ 3. 广州中国科学院沈阳自动化研究所分所 广州 511458)
}

\begin{abstract}
摘要: 针对水翼在波浪滑翔机运动中对本体产生的推进动力的预报问题, 利用 CFD 分析软件 Star-CCM+中的平面运动机构 (PMM) 模块, 对水翼翼板在波浪滑翔机主体随浪升沉过程中产生的升沉和绕自身旋转轴被动摆动的耦合运动过程进行三维模 拟。通过对运动过程中水翼产生的推进动力进行分析, 探究水翼的波浪参数(波高、周期)和结构参数(展弦比)等对水翼推进性 能的影响。研究发现, 在相同波高下, 水翼摆动角速度与波浪周期成反比; 水翼的前向平均推力系数与周期的平方成反比、 与波高值近似成二次项比例关系；展弦比增加可以增大翼板的平均推力系数，但增加幅度不大。
\end{abstract}

关键词: 波浪滑翔机; 三维水翼; 推进动力; 耦合运动; 波浪参数; 结构参数

中图分类号: TK79

\section{Study on Propulsion Performance of Three-dimensional Passive Swinging Hydrofoil on Wave Glider}



(1. State Key Laboratory of Robotics, Shenyang Institute of Automation,

Chinese Academy of Sciences, Shenyang 110016;

2. Institutes for Robotics and Intelligent Manufacturing, Chinese Academy of Sciences, Shenyang 110016;

3. Shenyang Institute of Automation, Guangzhou, Chineses Academy of Sciences, Guangzhou 511458)

\begin{abstract}
A three-dimensional simulation is carried out to analyze the coupled motion and hydrodynamic force of the hydrofoil, in order to predict the propulsion force generated by hydrofoils in the motion of wave glider. To model the wave-induced heave and passive swing of hydrofoil, the planar motion mechanism (PMM) module in CFD analysis software Star-CCM+ is used. By analyzing the propulsion force generated by hydrofoil during its motion, influences of wave parameters (wave height, period) and structural parameter (aspect ratio) on propulsion performance are investigated. Results of simulation show that under the condition of the same wave height, the angular velocity of hydrofoil is inversely proportional to the wave period. The forward average thrust coefficient of hydrofoil is inversely proportional to the square of the wave period, and increases quadratically with the wave height increasing. Average thrust coefficient is increased when increasing aspect ratio, but the effect is not very obvious.
\end{abstract}

Key words: wave glider; hydrofoil; propulsion force; coupled motion; wave parameters; structural parameters

\section{0 前言}

波浪滑翔机是一种有效利用波浪能和太阳能的 新型海洋观测型机器人, 具有续航能力长、负载能

* 国家重点研发计划(2017YFC0305804)、机器人学国家重点实验室自 主课题(2015-Z09)、中国科学院战略性先导科技专项子课题 (XDA22040101-02)和国家自然科学基金(41906173)资助项目。20190614 收到初稿, 20191127 收到修改稿
力强、可实时数据传输、清洁无污染等优点 ${ }^{[1-4]}$ 。 在波浪滑翔机的设计过程中, 波浪滑翔机前进动力 的主要来源是水翼, 水翼的水动力性能是影响波浪 滑翔机航行性能的主要因素之一。国内外学者对波 浪滑翔机及水翼的水动力特性进行了大量的研究 工作。田宝强等 ${ }^{[5-6]}$ 从能量转化角度建立了水翼与 上浮体耦合运动效率分析模型, 并计算和分析了 波高、波浪周期和翼板限位角对运动效率的影响; 桑宏强等 ${ }^{[7]}$ 和 $\mathrm{NGO}$ 等 ${ }^{[8]}$ 分别利用简化动力学方程进 
行理论分析的方法和利用海试数据进行概率预报模 型训练的方法, 均建立了波浪滑翔器纵向速度与海 浪参数间的定量关系; THAWEEWAT 等 ${ }^{[9]}$ 利用边界 元法建立了半自由摆动水翼的数值分析模型, 研究 了摆动频率和弹簧刚度对水翼摆动性能的影响; LIN 等 ${ }^{[10]}$ 、胡健等 ${ }^{[11]}$ 和 DAS 等 ${ }^{[12]}$ 利用计算流体力学方法 分析了三维主动摆动水翼的水动力特性, 分别得到 了不同运动方式、不同斯特罗哈尔数和不同摆动规 律下水翼的水动力性能; 吕元博等 ${ }^{[13]}$ 利用二维数值 模型对水翼的被动摆动过程进行了模拟, 探究了被 动旋转和主动旋转对 NACA0012 型水翼推力系数的 影响。

目前为至, 相关研究重点均集中在波浪滑翔 机的整体建模以及对水翼主动摆动水动力特性的 模拟, 而对被动运动的三维水翼推进动力的研究 还比较少见。本文针对波浪滑翔机及其水翼机构 特殊的运动方式与特点, 利用计算流体力学分析软 件 Star-CCM+中的平面运动机构 (Planar motion mechanism, PMM) 模块, 模拟三维水翼机构在波 浪滑翔机主体随浪升沉过程中产生的升沉和绕自 身旋转轴摆动的耦合运动过程, 并分析过程中水翼 产生的推进动力。探究了水翼翼板的结构主尺度参 数展弦比以及水翼运动参数限位角, 在不同波高和 波浪周期下对水翼推进性能的影响, 为水翼翼板的 设计以及波浪滑翔机在不同海况下的航行性能预 报提供参考。

\section{1 计算模型}

波浪滑翔机利用其上浮体带动水下滑翔体进 行升沉运动, 而水翼在垂直方向上的振荡运动和流 体的共同作用下, 产生绕自身轴的被动摆动, 进 而在水平方向上产生前向推力, 带动波浪滑翔机前 进 ${ }^{[14]}$ 。波浪滑翔机水翼机构的运动, 可以简化为垂 直面内升沉振荡和绕自身轴自由摆动的耦合运动。 本文利用 PMM 模块施加三维水翼模型一个垂向的 主动振荡运动, 并赋予其绕自身旋转轴被动旋转的 自由度。

波浪滑翔机水翼模型如图 1 所示, 水翼截面采 用常用的 NACA0012 翼型 ${ }^{[15]}$ 。波浪滑翔机随海面波 浪的升沉运动近似为垂向正弦振荡运动, 其振幅和 周期与海浪参数基本相同, 如式(1)所示

$$
\eta=A \sin \left(\frac{2 \pi}{T} t\right)
$$

式中, $\eta$ 为翼板垂向运动振幅; $A$ 为波高; $T$ 为波 浪周期。

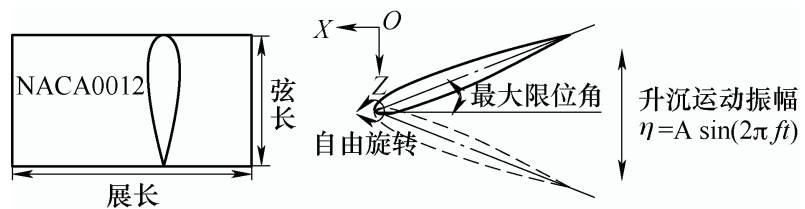

图 1 水翼及其运动模型

波浪滑翔机水翼产生的前向推力采用单位面积 下前向推力系数 $C_{\mathrm{T}}$ 来表示

$$
C_{\mathrm{T}}=\frac{T_{x}}{0.5 \rho b l}
$$

式中, $C_{\mathrm{T}}$ 为单位面积下前向推力系数; $T_{x}$ 为瞬时 前向推力; $\rho$ 为流体密度; $b$ 翼板弦长; $l$ 翼板展长。

波浪滑翔机特殊的运动方式导致了水翼翼板上 产生的前向推力系数是随着时间不断变化的。为了 便于比较, 将一个周期内水翼产生的前向推力系数 值对时间进行积分再除以作用时间, 得到水翼前向 的平均推力系数值。

$$
C_{\mathrm{Tav}}=\frac{1}{T} \int_{0}^{T} C_{\mathrm{T}} \mathrm{d} t
$$

式中, $C_{\mathrm{Tav}}$ 代表水翼产生的平均推力系数, 平均推 力系数越大, 水翼前向推力性能越好。



图 2 网格离散

采用重叠网格法模拟水翼的运动, 其 CFD 分析 模型的网格离散方式如图 2 所示, 其中深色网格部 分为背景网格区域, 浅色网格为重叠网格区域。背 景域前后、左右相距 40 倍弦长、上下相距 6 倍波高。 采用切割体网格对流域进行离散, 并在水翼升沉路 径附近进行网格加密处理。在水翼壁面处设置边界 层网格, 且保证壁面 $Y+$ 值小于 1 。计算选用雷诺平 均纳维-斯托克斯方程(RANS)和 $k-\omega$ 湍流模型, 并 采用 SIMPLE 方法求解分离流模型, 动量和湍动能 均采用二阶迎风格式求解。计算中保持背景域固定, 而重叠网格进行 DFBI(动态流体相互作用)运动, 并 启动 PMM 运动模块, 强迫水翼在垂向进行正弦升 沉运动, 同时放开水翼绕其自身旋转轴的转动自由 度。通过额外施加外力矩和编写自定义函数方式, 设置水翼转动限位角。 


\section{2 计算结果及分析}

影响波浪滑翔机水翼前向推力性能的主要因素 包括: 外界环境参数波浪的波高和周期、翼板结构 主尺度参数展长和弦长以及水翼摆动的限位角, 本 节主要针对各个参数对推力性能的影响进行探究。

\section{1 波浪周期对水翼前向推力性能的影响}

根据某海域波浪统计资料, 周期为 3 7 $\mathrm{s}$ 的海 况出现的频率相对较高。为了探究不同海浪周期下 波浪滑翔机水翼的前向推力性能, 分别选取波浪的 周期参数为 $3 s 、 4 s 、 5 s 、 6 s 、 7 s$ 和 $8 s$ 进行模拟 计算及分析。同时考虑到统计资料和计算量限制, 波浪的波高参数设为固定值, 水翼翼板摆动限位角 设为 $25^{\circ}$, 水翼翼板主尺度参数展弦比为定值。

模拟中, 滑翔机水翼运动的垂向振荡幅值和水 翼自由摆动的转角随时间的变化情况如图 3 所示。 从中可以看出, 当水翼垂向运动方向改变时, 翼板 迅速向另一侧摆动, 在极短的时间内达到最大限位 角附近。之后在阻尼力矩的作用下产生振荡并最终 趋于稳定摆角。同时, 可以看出在水翼垂向振荡运 动过程中, 水翼摆角值大多数时间内处于最大限位 角值附近。



图 3 水翼垂向振幅及摆角随时间变化关系

图 4 给出水翼翼板从波峰位置开始向下运动, 运动至波谷再回到波峰的运动轨迹, 及波浪周期内 的尾浴压力变化云图。从图 4 中可以看出, 当水翼 从波峰往波谷运动过程中, 翼板下表面为高压区, 而翼板上表面为负压区，并且不断有浴在前缘上部 生成、后移、脱落和耗散现象。当水翼运动到波谷 附近时, 速度降低, 翼板下表高压区逐渐消失, 并 出现在上表面。当水翼由波谷向波峰运动时, 翼板 向下摆动, 翼板上表面处于高压区, 下表面处于低 压区, 并且下表面存在浴的生成与脱落。在一个波 浪周期内, 翼板表面高压区始终位于水翼的后侧, 而低压区始终位于其前侧, 进而可以保证水翼产生
的水平方向的流体力始终向前。



图 4 单周期内水翼周围压力变化图

图 5 给出了波高为 $0.8 \mathrm{~m}$ 情况下，在单个垂荡 运动周期内，不同波浪周期下波浪滑翔机水翼的摆 角随时间的变化情况。为了便于对比，将摆角曲线 的时间横坐标均转化成自身周期的无量纲时间相对 值， $T$ 为自身波浪周期。通过对比可以发现，几组 波浪周期下水翼的摆角曲线几乎重合。

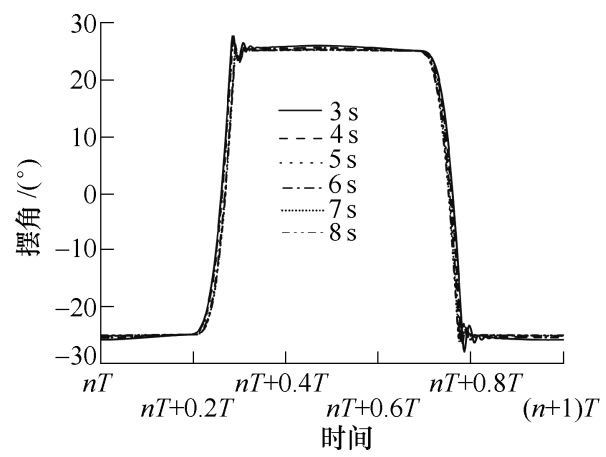

图 5 波高 $0.8 \mathrm{~m}$ 时不同波浪周期下水翼摆角随时间变化图



图 6 波高 $0.6 \mathrm{~m}$ 时不同波浪周期下水翼摆角随时间变化图

为了探究不同波高下摆角的变化规律，对波高 为 $0.6 \mathrm{~m}$ 和 $0.4 \mathrm{~m}$ 时不同周期下的水翼被动摆动进行 模拟，其摆角变化曲线如图 6 和图 7 所示，可以发 现在相同波高下摆角的变化规律完全相同。说明了 当波高一定时, 水翼在不同周期下被动摆动过程中， 
其过渡阶段和稳定摆角阶段占单个周期的时间比例 是相同的。同时也说明了水翼在过渡阶段的被动摆 动角速度与波浪周期是成反比的。

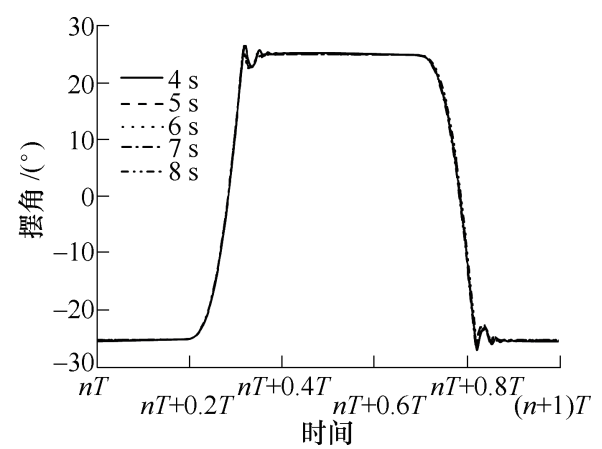

图 7 波高 $0.4 \mathrm{~m}$ 时不同波浪周期下水翼摆角随时间变化图

图 8 给出了在 $1 / 2$ 垂荡运动周期内 $($ 相对于各自 波浪周期的无量纲时间), 波高 $0.8 \mathrm{~m}$ 不同波浪周期 下波浪滑翔机水翼产生的前向推力系数随时间的变 化情况。可以看出, 水翼在被动摆动过程中可以产 生近似半正弦变化的前向推力。周期初始阶段中, 前向推力剧烈振荡过程对应水翼摆角变化曲线中摆 角达到其最大限位角的初期阶段。随着垂向运动方 向改变, 水翼从一侧摆向另一侧。当水翼摆到最大 限位角时, 会突然受到系统阻尼力矩的作用, 其角 加速度发生突变, 进而引起其转角和水动力性能在 短时间内的振荡。通过对比可以看出, 随着波浪周 期的减小, 虽然水翼摆角的振荡时间占周期的比例 基本没有变化, 但是其摆角稳定初始阶段推力的振 荡时间占自身周期比例增大。这是由于水平推力不 止与摆角、升沉运动有关系, 水体的惯性作用对推 力产生也有影响。同时, 波浪周期越小, 水翼产生 的瞬时前向推力越大。

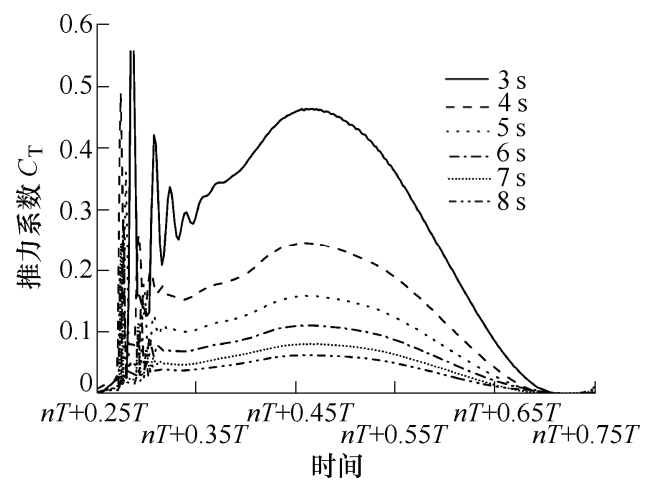

图 8 波高 $0.8 \mathrm{~m}$ 不同周期下水翼前向推力系数 随时间变化图

通过计算半个周期内水翼产生的平均推力系 数, 得到水翼前向平均推力系数与波浪周期间关系, 见图 9, 其水平轴坐标为周期平方的倒数。可以发 现，在同一波高下，水翼在各个波浪周期内产生的 前向平均推力系数值与波浪周期平方的倒数成正比
关系，且随着波浪周期的增大，水翼平均推力减小。

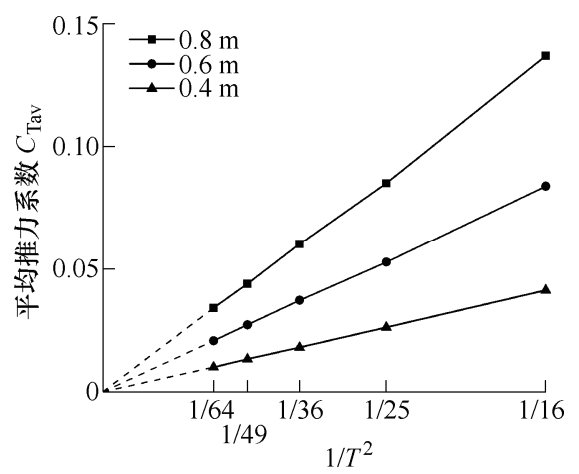

图 9 单位时间内水翼前向平均推力系数与波浪周期关系

\section{2 波高对水翼前向推力性能的影响}

根据某海域波浪统计资料, 波高为 $0 \sim 2.0 \mathrm{~m}$ 的 海况出现的频率相对较高。为了探究不同波高下波 浪滑翔机水翼的前向推力性能, 分别选取波高(振幅) 参数为 $0.2 \mathrm{~m} 、 0.4 \mathrm{~m} 、 0.6 \mathrm{~m} 、 0.8 \mathrm{~m} 、 1.0 \mathrm{~m} 、 1.2 \mathrm{~m}$ 和 $1.4 \mathrm{~m}$ 进行分析计算。同时考虑到计算时间成本, 波浪周期参数根据统计资料设为 $5 \mathrm{~s}$, 水翼翼板摆 动限位角设为 $25^{\circ}$, 水翼翼板主尺度参数展弦比 为定值。

图 10 给出了在单个垂荡运动周期内, 不同波 高下波浪滑翔机水翼的摆角随时间的变化情况。 可得, 随着波高(振幅)的增加, 水翼摆动速度加 快, 进而导致其在正负最大摆角间的过渡过程缩 短, 处于最大限位角位置的时间变长, 从而更有 效地转化波浪能为自身的前进动力, 能量转换效 率提高。

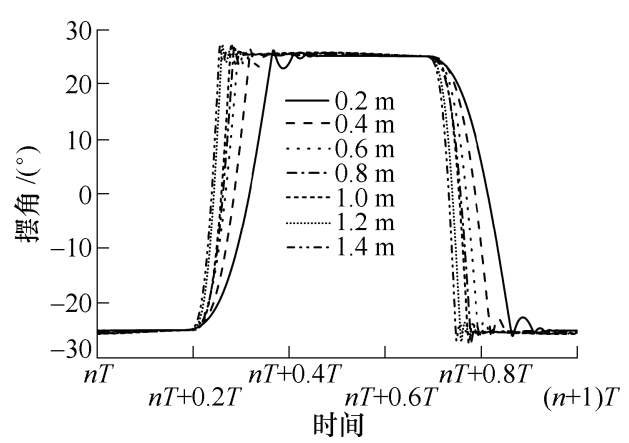

图 10 不同波高下水翼摆角随时间变化图

由图 10 可以得到翼板响应时间(翼板从一侧开 始摆动到另一侧摆角稳定)与波高间的关系, 见图 11 , 其纵坐标值代表了响应时间占波浪周期的比例。 随着波高增加, 纵坐标逐渐减小, 且减小趋势逐渐 变缓。通过对波高计算样本点的拟合, 发现对数函 数的拟合效果最好, 拟合曲线公式如式(4)所示

$$
T_{\mathrm{re}}=0.1171-0.085 \ln A
$$

式中, $T_{\mathrm{re}}$ 代表翼板摆角响应时间占波浪周期的比 例; $A$ 为波高。 


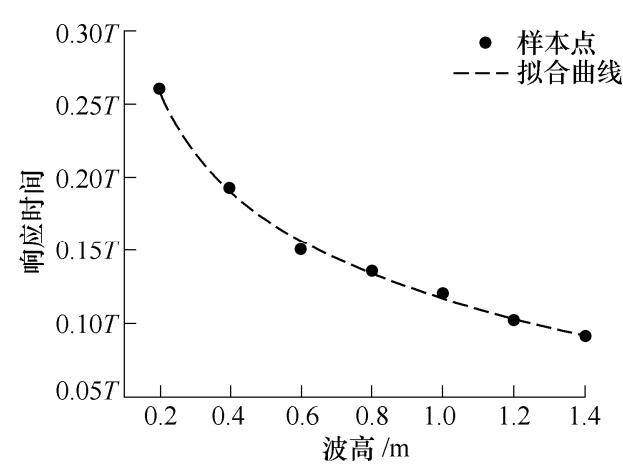

图 11 水翼翼板响应时间与波高关系



图 12 不同波高下水翼前向推力系数随时间变化图



图 13 水翼前向平均推力系数与波高关系

图 12 给出了在 $1 / 2$ 垂荡运动周期 $(2.5 \mathrm{~s})$ 内, 不 同波高下波浪滑翔机水翼产生的前向推力随时间的 变化情况。通过对比可知, 随着波高(振幅)的增加, 其初始振荡时间缩短。这是由于波高增大、垂荡振 荡速度加快, 导致流体阻尼力矩增大, 振荡时间减 小。随着波高的增加, 水翼产生的瞬时前向推力变 大。水翼产生的前向平均推力系数与波高的关系见 图 13。可以发现, 随着波高由小增大, 水翼产生的 前向平均推力系数先快速增大, 近似成二次项关系。 在波高增加到一定值后 $(1 \mathrm{~m})$, 水翼垂向振荡速度和 加速度值变大, 水翼周围流体运动剧烈表面产生空 泡现象, 导致其前向推力性能受到影响, 平均推力 系数随波高变化的增加量开始变缓。由第 2.1 节中 分析可知, 水翼平均推力系数与波浪周期的平方成 反比, 同时再考虑到本节中得到的平均推力系数与 波高的关系, 可得水翼平均推力系数与波浪周期及 波高的拟合公式如式(5)所示

$$
C_{\mathrm{Tav}}=\frac{2.25 A^{2}+0.85 A}{T^{2}}
$$

式中, $C_{\mathrm{Tav}}$ 代表水翼平均推力系数; $A$ 为波高; $T$ 为 波浪周期。

\section{3 翼板展弦比对水翼前向推力性能的影响}

为了确定波浪滑翔机水翼翼板的主尺度设计参 数值, 需要对不同展弦比参数下水翼的前向推力性 能进行探究。保持水翼翼板的面积不变, 分别选取 展弦比比值为 $3 、 4 、 5 、 6$ 和 7 的翼板进行分析计算。 其他参数分别设置如下: 波浪的波高设为 $0.8 \mathrm{~m}$ 、周 期 $5 \mathrm{~s}$ 、最大限位角为 $25^{\circ}$ 。

图 14 给出了在单个垂荡运动周期内, 展弦比参 数不同的水翼翼板的摆角随时间的变化情况。可以 看出, 几组摆角曲线几乎重合, 说明翼板展弦比参 数对翼板被动摆动过程的影响不大。图 15 给出了在 $1 / 2$ 垂荡运动周期内, 展弦比参数不同的水翼翼板产 生的前向推力系数随时间的变化情况。可知不同展 弦比下的水翼推力系数曲线基本重合，只是各推力 系数曲线的初始振荡阶段以及振荡结束至曲线峰值 间的过渡阶段略有不同。图 16 给出了水翼产生的平 均推力系数与翼板展弦比间的对应关系, 可以发现， 水翼产生的前向平均推力系数与翼板展弦比近似成 线性增长关系, 且平均推力系数随展弦比的变化值 不大。分析原因, 可能是因为展弦比增大, 水翼两 端面处产生的不规则浴旋影响变小, 从而使平均推 力系数有所增加。

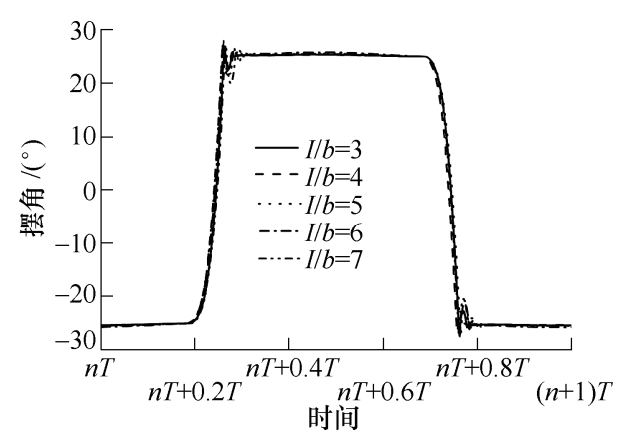

图 14 不同翼板展弦比下水翼摆角随时间变化图



图 15 不同翼板展弦比下水翼前向推力系数随时间变化图 


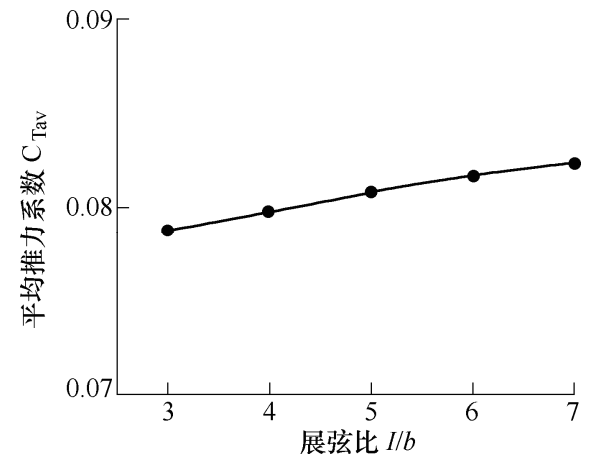

图 16 水翼前向平均推力系数与翼板展弦比关系

\section{4 限位角对水翼前向推力性能的影响}

波浪滑翔机水翼产生的前向推力与系统的最大 限位角密切相关。为了探究不同限位角下波浪滑翔 机水翼的前向推力性能, 分别选取系统限位角参数 为 $10^{\circ} 、 15^{\circ} 、 20^{\circ} 、 25^{\circ} 、 30^{\circ}$ 和 $35^{\circ}$ 进行分析 计算。其他参数分别设置为: 波浪的波高设为 $0.8 \mathrm{~m}$ 、 周期 $5 \mathrm{~s}$, 水翼翼板主尺度参数展弦比为定值。

图 17 给出了在单个垂荡运动周期内, 不同系统 限位角设定下波浪滑翔机水翼的摆角随时间的变化 情况。通过对比可知限位角越小, 其过渡阶段耗时 越小。图 18 给出了在 $1 / 2$ 垂荡运动周期内, 不同系 统限位角下波浪滑翔机水翼产生的前向推力系数随 时间的变化情况。通过对比可知, 在一定变化范围 内限位角越大, 水翼产生的瞬时前向推力最大值越 大，其推力系数曲线初始阶段的振荡时间也越长。 这是由于限位角越大, 水翼在达到限位角前时间越 长所需的动量越大, 停止摆动的时间越长。图 19 给出了水翼产生的前向平均推力系数与系统限位角 间的对比关系。可得水翼产生的前向平均推力系 数随着限位角的增大而增大, 并在 $25^{\circ}$ 后增大趋势 减缓。但是由于本文关于水翼前向推力性能的计 算过程中并没有考虑翼板在前进方向上的运动而 产生的阻力, 而只计算了水翼由于垂向振荡运动 而产生的前向推力性能, 进而导致了在限位角大 于 $25^{\circ}$ 后水翼推力系数还在增大的情况。在实际 中, 翼板限位角增大必然导致其前进方向的阻力 增大, 则水翼产生的净推力值(推力-阻力)随限位角

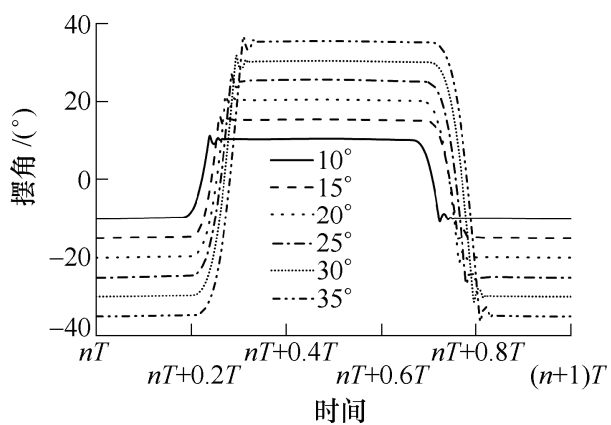

图 17 不同限位角下水翼摆角随时间变化图

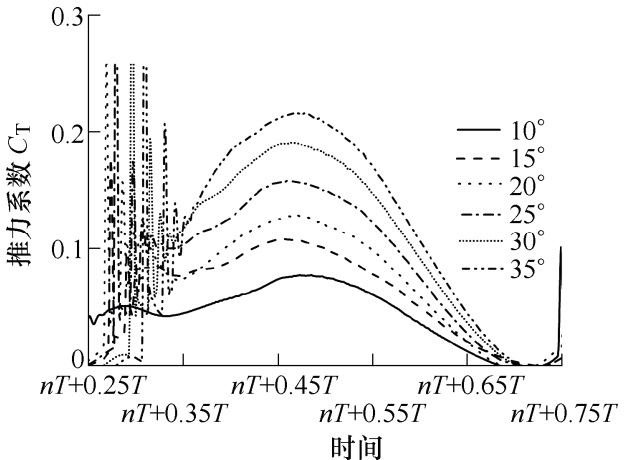

图 18 不同限位角下水翼前向推力系数随时间变化图

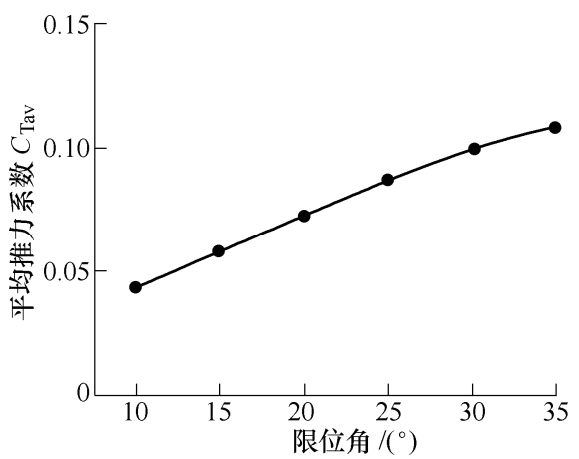

图 19 水翼前向平均推力系数与限位角关系图

变化曲线的极值点必然前移, 这一现象在我们将来 的研究中会着重考虑。

\section{3 结论}

利用 Star-CCM+中 PMM 模块对水翼在主动垂 向振荡运动下的被动摆动过程进行了模拟，通过对 不同波浪参数以及不同翼板主尺寸参数下水翼产生 的前向推力性能进行分析, 得到以下结论。

（1）相同波高，不同的波浪周期下，水翼摆角 相对波浪周期的变化曲线完全重合，水翼摆动角速 度与波浪周期成反比。水翼的前向平均推力系数与 周期平方的倒数成正比。

（2）波高越大，水翼在过渡阶段的被动摆动角 速度越快, 水翼产生的前向平均推力系数越大, 且 与波高值近似成二次项关系。

(3) 展弦比参数对翼板被动摆动过程的影响不 大。水翼产生的前向平均推力系数与展弦比近似成 线性增长关系，但变化幅值不大。

\section{参 考 文 献}

[1] 封锡盛, 李一平, 徐红丽. 下一代海洋机器人一写在 人类创造下潜深度世界记录 10912 米 50 周年之际 $[\mathrm{J}]$. 机器人, 2011, 33(1): 113-118.

FENG Xisheng, LI Yiping, XU Hongli. The next generation of unmanned marine vehicles-Dedicated to the 50 anniversary of the human world record diving 
10912 m [J]. Robot, 2011，33(1): 113-118.

[2] 陈质二, 俞建成, 张艾群. 面向海洋观测的长续航力移 动自主观测平台发展现状与展望 $[\mathrm{J}]$. 海洋技术学报, 2016, 35(1): 122-130.

CHEN Zhier, YU Jiancheng, ZHANG Aiqun. Overview on observation-oriented unmanned marine vehicles with high cruising ability: Development status and prospect [J]. Journal of Ocean Technology，2016，35(1): 122-130.

[3] 廖显雷, 李晔, 刘涛, 等. 波浪滑翔器技术的回顾与展 望[J]. 哈尔滨工程大学学报, 2016, 37(9): 1227-1236. LIAO Yulei, LI Ye, LIU Tao, et al. Unmanned wave glider technology: state of the art and perspective[J]. Journal of Harbin Engineering University, 2016, 37(9): 1227-1236.

[4] 俞建成, 孙朝阳, 张艾群. 无人帆船研究现状与展望 [J]. 机械工程学报, 2018, 54(24): 98-110.

YU Jiancheng, SUN Zhaoyang, ZHANG Aiqun. Research status and prospect of autonomous sailboats[J]. Journal of Mechanical Engineering, 2018， 54(24): 98-110.

[5] 田宝强, 俞建成, 张艾群, 等. 波浪驱动无人水面机器 人运动效率分析 $[\mathrm{J}]$. 机器人, $2014,36(1): 43-48,68$. TIAN Baoqiang, YU Jiancheng, ZHANG Aiqun, et al. Analysis on movement efficiency for wave driven unmanned surface vehicle[J]. Robot, 2014, 36(1): 43-48, 68.

[6] TIAN B Q, YU J C, ZHANG A Q. Lagrangian dynamic modeling of wave-driven unmanned surface vehicle in three dimensions based on the DH approach[C]//2015 IEEE International Conference on Cyber Technology in Automation, Control, and Intelligent Systems (CYBER). Piscataway, USA: IEEE, 2015: 1253-1258.

[7] 桑宏强, 李灿, 孙秀军. 波浪滑翔器纵向速度与波浪参 数定量分析 [J]. 水下无人系统学报, 2018, 26(1): 16-22. SANG Hongqiang, LI Can, SUN Xiujun. Quantitative analysis on longitudinal velocity and wave parameter of wave glider[J]. Journal of Unmanned Undersea Systems, 2018, 26(1): 16-22.

[8] NGO P, DAS J, OGLE J, et al. Predicting the speed of a wave glider autonomous surface vehicle from wave model
data[C]/2014 IEEE/RSJ International Conference on Intelligent Robots and Systems. Australian. IEEE, 2014: 2250-2256.

[9] THAWEEWAT N, PHOEMSAPTHAWEE S, JUNTASARO V. Semi-active flapping foil for marine propulsion[J]. Ocean Engineering, 2018, 147: 556-564.

[10] LIN X J, WU J, ZHANG T W. Performance investigation of a self-propelled foil with combined oscillating motion in stationary fluid[J]. Ocean Engineering, 2019, 175: $33-49$.

[11] 胡健, 赵旺, 王子斌. 三维摆动水翼仿生推进水动力分 析[J]. 应用科技，2019，46(2): 1-6.

HU Jian, ZHAO Wang, WANG Zibin. Hydrodynamic analysis of 3D flapping hydrofoil bionic propulsion[J]. Applied Science and Technology, 2019, 46(2): 1-6.

[12] DAS A, SHUKLA R K, GOVARDHAN R N. Foil locomotion through non-sinusoidal pitching motion[J]. Journal of Fluids and Structures, 2019， 89， 191-202.

[13] 吕元博, 田新亮, 李欣, 等. NACA 0012 摆动水翼水动 力特性的二维数值模拟 [J]. 中国舰船研究, 2018, 13(2): 7-15.

LÜ Yuanbo, TIAN Xinliang, LI Xin, et al. Two-dimensional numerical simulation of NACA 0012 flapping foil hydrodynamics[J]. Chinese Journal of Ship Research, 2018, 13(2): 7-15.

[14] MANLEY J, WILLCOX S. The wave glider: A persistent platform for ocean science[C]//OCEANS'10 IEEE SYDNEY. Piscataway, USA: IEEE, 2010: 1-5.

[15] ANDERSEN A, BOHR T, SCHNIPPER T, et al. Wake structure and thrust generation of a flapping foil in two-dimensional flow[J]. Journal of Fluid Mechanics, 2017, 812: R4-1-R4-12.

作者简介: 胡峰, 男, 1991 年出生, 硕士, 助理研究员。主要研究方向 为水下机器人水动力分析。

E-mail: hufeng@sia.cn

俞建成(通信作者), 男, 1976 年出生, 博士, 研究员, 博士研究生导师。 主要研究方向为新概念海洋机器人技术、移动自主海洋环境观测理论与 技术、海洋机器人智能控制方法与技术。

E-mail: yjc@sia.cn 